Canadian Journal of Applied Linguistics

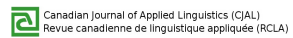

Revue canadienne de linguistique appliquée

\title{
Regard sur le développement de la compétence de communication à l'oral : récit rétrospectif d'un apprenant de français langue seconde
}

\section{Leif French, Suzie Beaulieu and Diane Huot}

Volume 20, Number 2, 2017

Special Issue: Current Perspectives on Oral Communicative

Competence of French Second Language Speakers

URI: https://id.erudit.org/iderudit/1042672ar

DOI: https://doi.org/10.7202/1042672ar

See table of contents

Publisher(s)

University of New Brunswick

ISSN

1920-1818 (digital)

Explore this journal

Cite this document

French, L., Beaulieu, S. \& Huot, D. (2017). Regard sur le développement de la compétence de communication à l'oral : récit rétrospectif d'un apprenant de français langue seconde. Canadian Journal of Applied Linguistics / Revue canadienne de linguistique appliquée, 20(2), i-xxiii.

https://doi.org/10.7202/1042672ar

Copyright (c) Leif French, Suzie Beaulieu, Diane Huot, 2017 Attribution 3.0 Unported (CC BY 3.0)

\section{cc) (i)}

This document is protected by copyright law. Use of the services of Érudit (including reproduction) is subject to its terms and conditions, which can be viewed online.

https://apropos.erudit.org/en/users/policy-on-use/ 


\title{
Introduction au numéro spécial
}

\section{Regard sur le développement de la compétence de communication à l'oral : récit rétrospectif d'un apprenant de français langue seconde}

\author{
Leif French \\ Sam Houston State University \\ Suzie Beaulieu \\ Université Laval \\ Diane Huot \\ Université Laval

\section{Introduction}

En guise d'introduction à ce numéro spécial, nous croyons important de faire le bilan de notre propre perspective entourant le thème central traité par l'ensemble des contributions du numéro. À cet égard, nous tenons compte dans cette introduction de nos expériences comme chercheurs, enseignants et apprenants pour tenter de faire état des multiples enjeux entourant le développement de la compétence de communication à l'oral dont nous avons été fréquemment témoins au fil des ans, et ce, dans divers contextes d'apprentissage du français. C'est précisément la présence de ces enjeux, qui sont en fait moins documentés dans la recherche auprès d'apprenants de français langue seconde (L2), qui nous a motivés à nous pencher sur le thème abordé dans ce numéro spécial.

Nous ne pouvons proposer un numéro spécial sur la compétence de communication à l'oral sans retracer brièvement l'évolution de cette notion en usage depuis maintenant 50 ans. L'importance et la diffusion de l'expression de compétence de communication sont attribuées à Hymes ${ }^{1}(1967,1972)$. Il a proposé que la compétence à communiquer ait reposé, tel que l'avait avancé Chomsky avec sa notion de compétence, sur des connaissances des structures de la langue ; cependant, il a ajouté que celles-ci, en soi, ne suffisaient pas pour permettre à un locuteur de communiquer avec efficacité. Selon Hymes (1972) il importait également de savoir quand se servir de ces structures en fonction de la situation pour atteindre ses objectifs communicatifs. Ainsi, Hymes (1972) a proposé une idée de compétence de communication qui revêtait en fait un caractère dynamique et variable. En effet, ce sont principalement les paramètres de la situation de communication et le lien entre les interlocuteurs de l'échange, et non des règles universelles et stables, qui balisent l'ensemble des productions langagières.

La présence de cette notion et la complexité qui s'y rattache se sont depuis répandues à d'autres domaines scientifiques, dont la pédagogie des langues. ${ }^{2}$ Dans les années 70, la notion de compétence de communication a été reprise pour combler le besoin d'adopter une vision de l'enseignement qui dépasserait la maîtrise des formes grammaticales. Que ce soit dans une perspective d'établissement de programmes d'études, de définition de contenu de cours, d'évaluation des apprentissages langagiers, on se préoccupait alors de trouver une façon d'organiser les connaissances requises pour 
développer une compétence de communication globale en sous-compétences ou composantes. Plusieurs modèles ou cadres sont issus de ces efforts. Parmi toutes les propositions, mentionnons les suivantes : Bachman et Palmer $(1996,2010)$; Canale et Swain (1980) ; Celce-Murcia (2007) ; Celce-Murcia, Dörnyei et Thurrell (1995) ; Conseil de 1'Europe (2001); Moirand (1982). En fait, toutes ces initiatives ont proposé un modèle qui, selon leurs perspectives respectives, rendait justice au caractère complexe et dynamique de la compétence à communiquer dans une L2.

En dépit des nombreuses descriptions de la compétence de communication publiées pendant toutes ces années, il est intéressant de noter que l'on trouve peu, à notre connaissance, de descriptions du développement de la compétence de communication de l'apprenant faites à partir de productions effectives de locuteurs, comme le suggérait initialement Hymes (1984). De même, à notre connaissance, il existe très peu de descriptions où l'apprenant aurait l'occasion de prendre la parole et de s'exprimer à propos de sa propre expérience, et ce, encore moins lorsque la langue cible est le français.

Ainsi, les lignes qui suivent proposent pour une première fois d'examiner en profondeur le développement de la compétence de communication à l'oral en L2 par le biais du récit rétrospectif d'un apprenant portant sur une période de plus de 20 ans. Pour ce faire, le récit sera commenté à l'aide du modèle multidimensionnel de Celce-Murcia (2007) dont on rappellera l'essentiel, après quoi d'autres facteurs affectant le parcours développemental de l'apprenant, et non associés au modèle retenu, seront évoqués. En guise de conclusion, nous proposerons une réflexion à l'égard du développement individuel de la compétence de communication en français L2.

\section{Développement de la compétence de communication à l'oral par le biais du récit d'un apprenant}

En dépit du grand nombre de modèles décrivant la compétence de communication en L2, et même si certains modèles, tels que ceux de Bachman et Palmer $(1996,2010)$ et de Canale et Swain (1980) ont connu une grande diffusion, le développement de la compétence de communication et le parcours de l'apprenant de ce récit seront décrits dans les termes d'un modèle général récent, à savoir celui de Celce-Murcia (2007, voir Figure 1). Ce choix se justifie par le fait que le modèle de Bachman et Palmer vise principalement la mesure et l'évaluation alors que le modèle de Celce-Murcia est plutôt lié à la pédagogie des langues. De plus, bien que le modèle de Canale et Swain concerne, lui aussi, la salle de classe, il ne prend pas en compte certains aspects de langue, dont l'utilisation d'expressions stéréotypées, qui selon les recherches récentes (voir Hilton, 2008 ; Wood, 2009) contribuent de façon importante à la compétence de communication. Le modèle de Celce-Murcia accorde une place d'importance aux expressions stéréotypées sous la forme de compétence « formulaïque » (ou compétence liée à l'emploi de formules et d'expressions stéréotypées). 


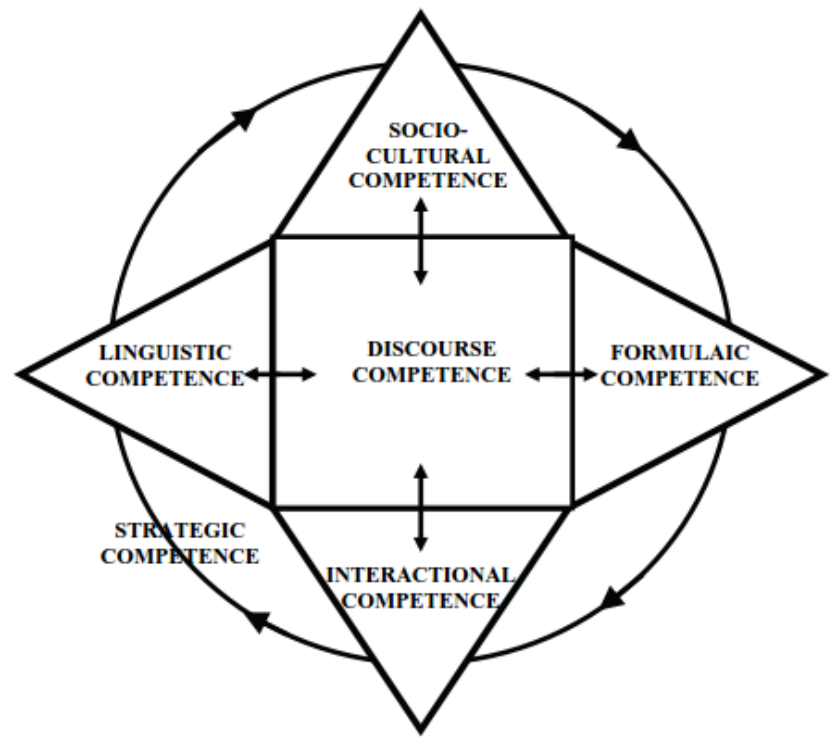

Figure 1. Modèle de compétence de communication de Celce-Murcia (2007).

De plus, l'intérêt du modèle de Celce-Murcia (2007) vient principalement de sa vision à caractère dynamique de la compétence de communication, qui se rapproche davantage de la définition initiale proposée par Hymes (1972) dans le sens où on reconnaît que les différentes parties du modèle interagissent les unes avec les autres (voir Tableau 1). Par exemple, la compétence socioculturelle et la compétence interactionnelle constituent deux facettes d'un même construit, la première représentant des éléments du haut vers le bas (top-down elements) et la seconde, du bas vers le haut (bottom-up), ceci pour l'ensemble des connaissances nécessaires pour s'exprimer de façon appropriée dans un contexte et une culture donnée. Il en va de même pour les compétences grammaticale et formulä̈que, ces deux composantes combinées contribuant, toujours selon cette auteure, à la production d'énoncés à la fois précis et fluides. C'est la mise à contribution de ces quatre éléments ou compétences qui permet, selon elle, à la compétence discursive de se déployer. En ce qui concerne la compétence stratégique, elle vient pallier des faiblesses dans une des compétences. Bref, ce modèle, à notre avis, permet donc de mieux tenir compte des complexités linguistiques et socioculturelles qui risquent de se manifester chez l'apprenant à différents stades du développement de sa compétence à l'oral.

Le Tableau 1 résume chacune des compétences du modèle de Celce-Murcia (2007). Pour chaque composante de la compétence de communication, on y trouve des précisions sur la définition, sur les connaissances requises pour développer chacune des composantes et sur des parallèles avec les autres modèles connus. 
Tableau 1

Résumé du modèle de Celce-Murcia (2007)

\begin{tabular}{|c|c|c|c|}
\hline Compétences & Définition & $\begin{array}{l}\text { Connaissances de la langue cible } \\
\text { requises }\end{array}$ & $\begin{array}{l}\text { Parallèles avec d'autres modèles fréquemment } \\
\text { cités }\end{array}$ \\
\hline Linguistique & Connaissances de la langue système & $\begin{array}{l}\text { - Connaissances phonologiques } \\
\text { - Connaissances lexicales } \\
\text { - Connaissances morphologiques } \\
\text { - Connaissances syntaxiques }\end{array}$ & $\begin{array}{l}\text { - Compétence linguistique (Celce-Murcia et } \\
\text { coll., 1995; Chomsky, 1957, } 1965 \text {; Hymes, } \\
\text { 1967, 1972) } \\
\text { - Compétence grammaticale (Bachman et } \\
\text { Palmer, 1996, } 2010 \text {; Canale et Swain, 1980) }\end{array}$ \\
\hline Formulaïque & $\begin{array}{l}\text { Connaissances des segments de langue } \\
\text { figés et préétablis que le locuteur } \\
\text { emploie fréquemment lors } \\
\text { d'interactions quotidiennes }\end{array}$ & $\begin{array}{l}\text { - Routines } \\
\text { - Cooccurrences } \\
\text { - Expressions idiomatiques } \\
\text { - Expressions stéréotypées } \\
\text { - Paramètres lexicaux }\end{array}$ & \\
\hline Socioculturelle & $\begin{array}{l}\text { Connaissances des éléments servant à } \\
\text { s'exprimer de manière appropriée à } \\
\text { l'intérieur d'un contexte social et } \\
\text { culturel donné }\end{array}$ & $\begin{array}{l}\text { - Variation sociostylistique } \\
\text { - Normes socioculturelles } \\
\text { - Normes pragmatiques }\end{array}$ & $\begin{array}{l}\text { - Compétence communicative (Hymes, } 1967 \text {, } \\
\text { 1972) } \\
\text { - Compétence sociolinguistique (Bachman et } \\
\text { Palmer, 1996, 2010; Canale et Swain, } 1980 \text {; } \\
\text { Celce-Murcia et coll., 1995) }\end{array}$ \\
\hline Interactionnelle & $\begin{array}{l}\text { Connaissances des habiletés } \\
\text { conversationnelles nécessaires pour } \\
\text { respecter les conventions d'usage quant } \\
\text { aux formules de politesse et de } \\
\text { coopération et aux stratégies pour } \\
\text { ménager la face. }\end{array}$ & $\begin{array}{l}\text { - Actes de paroles } \\
\text { - Conventions de tours de } \\
\text { paroles/prise de parole } \\
\text { - Indices non verbaux et } \\
\text { paralinguistiques }\end{array}$ & $\begin{array}{l}\text { - Compétence illocutoire (Bachman et Palmer, } \\
\text { 1996, 2010) } \\
\text { - Compétence actionnelle (Celce-Murcia et } \\
\text { coll., 1995) }\end{array}$ \\
\hline Discursive & $\begin{array}{l}\text { Connaissances de la façon dont les } \\
\text { mots, les structures et les énoncés } \\
\text { s'agencent pour produire un message } \\
\text { cohérent à l'oral ou à l'écrit }\end{array}$ & $\begin{array}{l}\text { - Cohésion } \\
\text { - Deixis } \\
\text { - Cohérence } \\
\text { - Structure générique du texte }\end{array}$ & $\begin{array}{l}\text { - Compétence discursive (Canale et Swain, } \\
\text { 1980) } \\
\text { - Compétence textuelle (Bachman et Palmer, } \\
\text { 1996, 2010) }\end{array}$ \\
\hline Stratégique & $\begin{array}{l}\text { Connaissances des comportements ou } \\
\text { des mécanismes de réflexion servant à } \\
\text { promouvoir l'apprentissage ou la } \\
\text { communication }\end{array}$ & $\begin{array}{l}\text { - Stratégies cognitives } \\
\text { - Stratégies métacognitives } \\
\text { - Stratégies de mémorisation } \\
\text { - Stratégies de communication }\end{array}$ & $\begin{array}{l}\text { - Compétence stratégique (Bachman et Palmer, } \\
\text { 1996, } 2010 \text {; Canale et Swain, } 1980 \text {; Celce- } \\
\text { Murcia et coll., 1995) }\end{array}$ \\
\hline
\end{tabular}




\section{Présentation de l'apprenant}

L'apprenant de cette étude, le premier coauteur de cet article, ci-après LMF, est un adulte dans sa quarantaine, locuteur natif de l'anglais, né aux États-Unis et ayant fait des études primaires et secondaires dans ce même pays.

Il a commencé l'étude du français L2 aux États-Unis. De la $7^{\mathrm{e}}$ année à la $12^{\mathrm{e}}$ année, il a suivi des cours basés sur l'approche audiolinguale à raison d'une heure, deux fois par semaine. À la fin de ses études secondaires, il avait atteint un niveau intermédiaire bas, selon l'échelle de l'American Council on the Teaching of Foreign Languages (ACTFL). Par la suite, à l'âge de 19 ans, il a déménagé en Europe où il a fait un diplôme d'études françaises dans une université de l'ouest de la France. Puis, il a poursuivi des études de premier cycle en linguistique française à la fois à l'Université de Paris et à la California State University. Pendant cette période, il a également travaillé régulièrement dans une banque et dans l'industrie du tourisme dans le sud de la France. Finalement, à l'âge de 26 ans, il s'est établi au Québec, dans la région du Saguenay, pour poursuivre des études de $2^{\mathrm{e}}$ et $3^{\mathrm{e}}$ cycles en dialectologie et en psycholinguistique, respectivement. Après ses études doctorales, il a obtenu un poste de professeur en linguistique appliquée dans une université québécoise. LMF présente ici certains événements qui ont, selon lui, marqué de façon considérable le développement de sa compétence à communiquer en français. Tout au long de son parcours d'apprenant/usager du français, LMF a tenu un journal de bord dans lequel il notait régulièrement ses observations et ses expériences par rapport à son apprentissage du français. Il portait surtout attention aux enjeux rattachés à la communication à l'oral et, plus particulièrement, aux éléments qui nuisaient à ou favorisaient la réussite de ses interactions avec des locuteurs natifs. Il faut souligner que ses entrées n'ont pas toujours été écrites de façon systématique, détaillée ou à intervalles réguliers au fil du temps. Certains événements ont été immédiatement inscrits au journal alors que d'autres l'ont été après un délai de quelques jours, voire quelques mois.

Le récit, dont l'intégralité se retrouve en annexe, a été rédigé de façon rétrospective en 2014. Il a servi de mot d'introduction au colloque Perspectives actuelles sur la compétence communicative à l'oral en français $L 2$ organisé dans le cadre du congrès de l'Association francophone pour le savoir (ACFAS). Les exemples se trouvant dans le récit ont été puisés dans le journal de bord en fonction des sujets abordés dans les communications retenues pour le colloque. Étant donné sa formation universitaire et ses champs d'intérêt de recherche, on comprendra que le regard rétrospectif de LMF diffère vraisemblablement de celui d'un apprenant naïf; LMF s'exprime principalement à l'aide de descriptions linguistiques et paralinguistiques détaillées, propres à un expert en acquisition des L2.

Nous reconnaissons les problèmes d'ordre méthodologique que peuvent comporter les données auto déclarées de LMF, et ce, principalement en ce qui concerne la fiabilité avec laquelle il rapporte les événements à travers le temps et la généralisation de ses observations. Cependant, les déclarations de LMF fournissent un outil indéniable pour documenter le caractère dynamique, complexe et individuel du développement des différentes composantes du modèle de Celce-Murcia (2007), une perspective sur l'apprentissage du français pour laquelle il existe très peu de recherches. 


\section{Le récit de l'apprenant : partie 1}

La présentation et la discussion du récit de cet apprenant comportent deux parties : (a) la compétence de communication : de débutant à avancé et (b) la compétence de communication : l'usager compétent et son rendez-vous avec la variété québécoise. Le développement de la compétence de communication et le parcours de l'apprenant seront décrits dans les termes de Celce-Murcia (2007) dans la section qui suit.

\section{Prise de conscience 1 : «Ce qu'on apprend n'est pas toujours ce qu'on}

entend ! » Dans la première partie du récit, LMF a décrit les premiers moments de son apprentissage du français aux États-Unis. On constate, quand LMF a commencé son apprentissage guidé, qu'il était exposé à un certain input en classe (voir les extraits 1 et 2). Grâce à l'enseignement proposé, LMF a travaillé au développement de certains éléments de la compétence formulaïque. De plus, comme l'apprenant a évoqué la répétition d'actes de parole et la forme d'activités (ici de type question-réponse), on peut aussi penser que LMF semblait travailler certains aspects de la compétence interactionnelle, telle que décrite par Celce-Murcia (2007, p. 48).

1 Notre enseignante nous faisait répéter continuellement des énoncés basés sur un acte de parole. Par exemple, elle nous disait « Bonjour classe. Comment allez-vous ? » . . nous répondions, comme de petits robots « Je vais bien! Et vous?»

2 «Comment vous appelez-vous ? » ou « Comment t'appelles-tu ?» Une question à laquelle on répondait rapidement et avec fierté : « Je m’appelle . . . »

On notera que bien qu'on ait tenté de développer la compétence formulaïque et la compétence interactionnelle, dans les faits, les énoncés enseignés et pratiqués en classe ne représentaient pas l'usage fréquent (p. ex. selon Guérin [2011], l'interrogation formulée à l'aide de l'inversion est peu utilisée par des locuteurs natifs dans leurs interactions quotidiennes). Il y manquait aussi, comme c'était souvent le cas à l'époque de l'audiolinguale, des précisions sur la valeur sociolinguistique de ces énoncés.

De plus, les extraits 3 et 4 ont révélé que LMF travaillait en parallèle certains aspects de la compétence linguistique. Les activités proposées lui donnaient l'occasion d'amorcer le développement de certaines connaissances d'ordre phonétique ou d'ordre syntaxique, liées à l'ordre des mots.

3 Il y avait aussi des exercices de répétitions incessants pour travailler non seulement la «bonne » prononciation.

4 ... mais aussi, pour nous sensibiliser à l'ordre des adjectifs en français. Donc, toute la classe chantait souvent en cœur : « un crayon ! Bleu . . . un crayon bleu !» « Gros . . . un gros crayon! » « Un gros crayon bleu ! »

Enfin, amené un jour à mettre à l'épreuve ses connaissances en interagissant avec des locuteurs natifs au Consulat de France de San Francisco, LMF a découvert que les 
éléments appris en classe ne suffisaient pas pour lui permettre d'interagir avec efficacité, même lors des échanges les plus rudimentaires avec des locuteurs natifs du français :

5 Par contre, vous pouvez vous imaginer la totale déception linguistique vécue lors de notre première activité culturelle au Consulat de France à San Francisco, quand les jeunes étudiants français en échange nous tendaient chaleureusement la main en disant : «Salut, ça va ? C'est quoi ton nom ? », ou bien la confusion totale à une question simple posée par l'animateur de l'activité : «Toi, t'es dans quel bus? Le gros bus jaune ?»Question à laquelle on se demandait s'il fallait répondre en précisant : «Oui, le bus jaune, le gros bus, le gros bus jaune. »

En ce qui concerne la description de l'événement présenté à l'extrait 5, selon le modèle de Celce-Murcia (2007), on peut penser qu'il s'agissait d'un premier contact non guidé avec des variantes informelles de la langue française, lesquelles constituent une connaissance liée au développement de la compétence socioculturelle, une fois que sont comprises les valeurs sociolinguistiques qui leur sont associées. Il est impossible de savoir avec certitude si la compétence socioculturelle de LMF s'est développée grâce à cette situation, mais on peut formuler l'hypothèse que l'apprenant a découvert que la langue en salle de classe différait sensiblement de ce que les gens utilisent en contexte naturel. Bien que cette prise de conscience n'ait pas forcément affecté le développement de l'oral proprement dit, cela a pu avoir sensibilisé l'apprenant au fait que les contextes de communication avec des locuteurs natifs pouvaient nécessiter une modification de la langue apprise en classe.

Par la suite, on apprend qu'ayant quitté les États-Unis, LMF a poursuivi son apprentissage du français à Angers, dans l'ouest de la France, où il a suivi, entre autres, un cours de phonétique avec des locuteurs natifs du français.

6 Tout fier d'être inscrit au cours de phonétique avec des locuteurs natifs, je travaillais très fort afin d'assimiler toutes les nuances acoustiques de la langue française. Surtout, je passais des heures au laboratoire de langue pour bien percevoir et bien réaliser la distinction phonétique entre la première personne au singulier au futur et celle du conditionnel, comme dans je prendrai /e/ versus je prendrais /e/.

On note à l'extrait 6 que LMF a continué à développer sa compétence linguistique à l'oral, en particulier ses connaissances phonologiques et phonétiques. Il a découvert cependant un phénomène important relié à la neutralisation de l'opposition des phonèmes /e/ et $/ \varepsilon /$. Alors qu'il apprenait dans ce cours de phonétique que le /e/ et le / $/ \varepsilon /$ constituaient deux phonèmes distincts, il faisait un constat différent à l'extérieur de la classe, ce qui l'a porté à s'interroger. LMF semblait remettre en question sa capacité de percevoir cette distinction, alors que l'enseignant ou les membres de la communauté ne la pratiquaient pas. Pour la deuxième fois, LMF a manifestement constaté l'écart entre ce qui était enseigné et ce qui était utilisé par les locuteurs natifs dans des contextes de communication de vie réelle.

En somme, à ce premier stade de son apprentissage du français décrit dans les termes de Celce-Murcia (2007), il semble que soit amorcé le développement de certaines 
composantes des compétences formulaïque, interactionnelle et linguistique en contexte guidé. Également, ses premières expériences avec des locuteurs natifs ont peut-être éveillé en lui une certaine conscience sociolinguistique, ce qui aurait ainsi contribué au développement de sa compétence socioculturelle (voir les travaux de Chevrot, Nardy, Barbu [2011] en français langue maternelle à ce sujet).

Prise de conscience 2 : «À l'oral, les hésitations ou le silence ne sont pas bons ! » Dans la deuxième section de la première partie du récit, LMF a expliqué à l'extrait 7 comment il a eu recours à des stratégies afin de démontrer à ses interlocuteurs qu'il avait une compétence de communication élevée.

7 La gloire de la victoire est finalement arrivée! Toujours époustouflé par les réactions imprévues de mon interlocuteur, je me suis embarqué dans une quête linguistique afin de réduire et de, potentiellement, éradiquer le silence et les hésitations irrégulières de mon parler. Conclusion préliminaire de l'enquêtec'était un exploit impossible ! Par contre, une découverte valant de l'or a émergé de cette quête : une nouvelle stratégie de communication me permettait de briser le silence et de camoufler les hésitations. Celle-ci consistait tout simplement à saupoudrer ma parole d'interjections lexicales stratégiques comme : ben ... bon ben ... t'sais ou bien à utiliser la petite pause euh, mais — attention — soigneusement réalisée à la française comme euh et non pas umm ... ou ahh ... !

Exprimée dans les termes de Celce-Murcia (2007), cette expérience relatée par l'apprenant laisse voir que LMF utilisait des stratégies d'apprentissage métacognitives lorsqu'il faisait une auto-évaluation de son succès conversationnel. Il a identifié des solutions pour pallier les lacunes qu'il avait identifiées dans sa compétence interactionnelle et, principalement, sa compétence conversationnelle (Celce-Murcia, 2007, p. 49-50). Parmi les solutions trouvées, il utilisait une stratégie de communication pour gagner du temps et prenait aussi soin d'éliminer des traces de prononciation appartenant à l'anglais, le tout dans un effort de rapprocher son parler de celui des locuteurs natifs. Grâce à ces modifications, LMF espérait faciliter la compréhension de ces interlocuteurs.

Poursuivant sa réflexion à propos du développement de sa compétence de communication à l'oral, LMF a raconté qu'il avait eu l'occasion de travailler dans une banque où il a découvert qu'au moment où il communiquait avec les clients, ces derniers se penchaient de moins en moins vers lui pour essayer de le comprendre (voir l'extrait 8).

8 Soudainement, je me suis rendu compte que lorsque je communiquais avec les clients, ils ne se penchaient plus que rarement vers moi avec ces fameuses grimaces de confusion et d'incertitude que j'avais pris l'habitude de voir chez mes interlocuteurs.

À partir de ces observations, il en a donc conclu que la règle qu'il avait déduite, celle de maîtriser le phénomène de pausologie et d'hésitations pour paraître compétent à l'oral, semblait fonctionner. On peut penser que LMF avait atteint une telle maîtrise de ces stratégies de communication, qu'il était alors passé à une étape supérieure et qu'il semblait maintenant avoir développé une composante de la compétence interactionnelle. 
Ainsi, la première partie du récit de l'apprenant s'est terminée avec les efforts de LMF pour développer sa compétence interactionnelle. De plus, puisque les locuteurs natifs ne modifiaient plus leur comportement non verbal en sa présence, il a jugé que sa compétence en français était maintenant avancée et qu'il avait la légitimité de se considérer comme étant un bilingue (voir l'extrait 9).

9 Enfin, j'avais atteint le statut de membre distingué du Club des bilingues, celui qui semblait être certifié ou, tout du moins, approuvé par les locuteurs natifs !

Il est intéressant de noter qu'il a créé sa propre définition de «bilinguisme » qui était basée sur la perception de sa capacité à livrer un message intelligible et compréhensible à son interlocuteur. Notons qu'il s'agissait d'une définition peu théorique ou politique, mais plutôt d'une définition basée sur sa propre expérience en tant qu'usager de français évoluant parmi des francophones.

\section{Le récit de l'apprenant : partie 2}

La deuxième partie du récit présente la vie de LMF dans la belle province avec tout ce que ce changement de continent comporte sur le plan des expériences interactionnelles et linguistiques. L'analyse de cette partie sera effectuée en deux temps, correspondant à deux grandes prises de conscience qui ont influencé son parcours d'usager du français L2.

Prise de conscience 3 : « Le français parlé au Québec bat au rythme d’une chanson bien différente. " Cette partie du récit de LMF contient un grand nombre d'observations relatives à sa vie dans la province de Québec, plus précisément dans la région du Saguenay, située sur la rive nord du Saint-Laurent. Le parler français de cette région est caractérisé notamment par des particularités d'ordre phonétique et lexical qui se distinguent du parler auquel il avait été exposé précédemment en France. Une certaine adaptation à une nouvelle variété de français lui a ainsi été nécessaire. C'est pourquoi plusieurs remarques formulées dans cette partie du récit concernaient la prononciation et le lexique propres à cette région (voir les extraits 10,11 et 12).

10 Lors de mon arrivée au Saguenay, appelé de manière populaire le «Royaume du nord », je débarque dans le petit aéroport de Bagotville et me dirige au comptoir de la « location d'autos » (et non-bizarrement—la «location de voitures »).

11 Là, un monsieur d'un certain âge m'attendait avec un sourire chaleureux : «Bonhour Cher! » Pas trop certain ni de la prononciation de bonhour ni de la signification de Cher, je ne me laisse pas déconcerter par l'incertitude lexicale-phonologique et je lui adresse un «Bonjour Monsieur » bien articulé!

12 Ensuite, il se tourne vers moi et en me regardant au-dessus de ses petites lunettes d'un air curieux, il dit : «Cou'don! Qu'esse tsu vient faire dans c'coin icitte ?! » Je suis resté bouche bée !!!! Grâce à son intonation 
montante, je savais qu'il venait de me poser une question, mais, à ma grande surprise, je n'avais pu capter que deux mots : faire et coin.

En plus d'être intrigué par cette nouvelle variété de français, il a fait face à certains défis lorsqu'il tentait de se faire comprendre alors qu'il utilisait une variété de français qui différait de celles en usage au Saguenay :

13 Muni d'une mine de connaissances couronnées par l'Académie française et le Grevisse, je planifie soigneusement ma phrase pour qu'elle sonne précise, compétente et intelligible : «S'il vous plait, pourriez-vous m'indiquer où se trouve le boulevard Talbo ?» Le chauffeur a hésité un instant : «Ah ... tsu veux dire le boul'vard (ord) Talbo(t)! Garde ! c'st ben facile . . . tsu vas tout drète-là ! Pis . . . tsu fais un ptsi boutte . . . pis tsu vires à gauche-là ! Pis, là ! là ! Tsu fais un autr' boutte un peu plus loin ... pis tsu va l'voér. » À ma grande déception, je n'ai rien compris cette fois-là !! Tout ce je pouvais déchiffrer de cet échange c'était un enchainement de pi-tsu-là, pi-tsu-là, boutte! pi-tsu-là, boutte! En fait, il a fallu deux jours de recherche dans les ouvrages de dialectologie avant de bien saisir le sens de boutte et de comprendre que le bourdonnement des sons $(t s u, t s u, d z u)$ était mon premier cours 101 sur le phénomène de l'affrication au Québec.

Ainsi, dans cette première section de la deuxième partie du récit, on constate que ces faits relatés dans les termes de Celce-Murcia (2007) appartiennent principalement à la compétence socioculturelle en raison de la variation linguistique. De plus, la rencontre avec la variété saguenéenne a semblé mener LMF à une remise en question de sa compétence bilingue et de l'étendue des connaissances qu'il avait acquises.

\section{Prise de conscience 4 : « Je m'aligne-tu au français du Québec et de ma}

région ? » La dernière partie du récit est dominée par des questionnements liés à la maîtrise d'éléments appartenant à la variation sociolinguistique et, principalement, à la variation phonétique. LMF a continué de développer sa compétence socioculturelle en se familiarisant avec les variantes en usage dans son nouveau milieu de vie. Son apprentissage allait au gré des hypothèses qu'il émettait face aux nouvelles formes qu'il rencontrait et à sa grande volonté d'intégrer ces formes à son répertoire sociolinguistique (voir l'extrait 14).

14 Il y avait aussi mes sorties de pêche où j'ai fait la découverte d'une drôle de forme linguistique. Par exemple, mes «chums » de pêche avaient l'habitude d'employer fréquemment $t u$ ou $t i$ dans les questions comme « Ça s'peuttsu ? », « Ça mord-tsu ? », « T'as-tsu une bière ? » ou encore « Ton couteau, ça coupe-tsi comme y faut?». J'étais très intrigué par la complexité linguistique de ce phénomène et je me suis donc mis à créer et à pratiquer mes propres phrases avec $t s u$.

Malgré ses efforts pour se familiariser avec les usages locaux, LMF a perçu que ses interlocuteurs le considéraient comme un membre périphérique de leur communauté linguistique (selon les termes de Lave et Wenger, 1991). Il s'est alors demandé quelles 
stratégies il devait mettre en place pour qu'un jour on lui confère le statut de membre légitime. Les deux extraits suivants (15 et 16) illustrent bien cette situation.

15 À un moment de la conversation, il m'a arrêté en riant : «T'es donc ben drôle ! Pourquoi tu dis tout le temps applon? Nous-autres, on dit pas ça de même ! C'est un appelin !». Quelle intervention puissante provenant d'un ami forestier non-linguiste ! Mais aussi quelle révélation personnelle ! Dans la petite phrase de mon ami «nous-autres, on dit pas ça!», on m'avait très curieusement placé à l'extérieur du pronom nous-autres et, pourtant, cela faisait déjà plus d'un an que je restais au Québec. Est-ce que je n'étais pas intégré ?!

16 Est-ce même possible d'être inclus ? Si oui, sur quels indices de l'oral devraisje me baser pour faire partie du club des «nous-autres »? Après tout, ni l'Académie française, ni l'Office de la langue française au Canada, ne semblaient être trop certains quant au chemin à prendre pour obtenir un abonnement au «Club des nous-autres » au Québec.

En faisant de nouveau appel à sa compétence stratégique, LMF a formulé l'hypothèse qu'il devait maîtriser les phénomènes de variation phonétique locaux s'il voulait ne plus être perçu comme étranger au groupe. En d'autres mots, il a conclu qu'il devait développer davantage sa compétence socioculturelle et a fait le choix conscient de consacrer entièrement son attention à cet élément de la compétence de communication à l'oral.

17 En fait, je suis devenu un véritable laboratoire linguistique mobile et je testais constamment mes hypothèses en fonction de mes observations minutieuses. J'ouvrais certaines voyelles pour être certain de bien réaliser les prononciations comme « chal/E/ » (chalet), «1/E/» (lait), ou bien « pa » (pas) et « po » (le gros «a » dans pas) et ensuite je lisais attentivement la réaction de mon interlocuteur ou je leur demandais carrément leur impression de mon usage. Lorsque je ressentais une impression négative ou un jugement quelconque, j'en prenais note mentalement.

Par ailleurs, LMF a dû se rendre à l'évidence qu'il ne suffisait pas de comprendre la formation ou la réalisation des variantes locales auxquelles il était fréquemment exposé pour pouvoir ensuite les utiliser avec succès. Il lui fallait comprendre que ces formes s'utilisaient en fonction des paramètres de la situation de communication et que la transgression, même involontaire, des normes d'usage implicites pouvait nuire au succès de ses interactions (voir l'extrait 18). Il lui fallait également comprendre que même s'il saisissait les nuances pragmatiques de ces variantes, on pouvait tout de même lui en refuser l'usage (voir l'extrait 19) :

18 Un jour, une technicienne en informatique me demande mon numéro de matricule pour remplir une demande et, impatient de démontrer à mon entourage ma compétence de l'emploi de $t u$, je lui ai rapidement répondu : «Je sais-tsu moé ?» Sa réponse fut complètement inattendue : « Ah, 
Monsieur est un peu baveux à matin ! ' Évidemment, dans tout mon effort de maîtriser l'emploi structural de la petite particule $t u$, j'étais malheureusement passé à côté de certaines nuances pragmatiques assez importantes !

19 Je dois avouer qu'avec ma méthodologie, cela n'a pas toujours été évident de bien discerner la norme d'usage. Par exemple, un jour, j'ai lâché un gros «MARCI ! » devant une amie et sa réaction a été instantanée et tranchante : «T'sais . . . c'est vraiment pas beau de dire ça! C'est les gens peu scolarisés de rang qui disent ça !» J'ai trouvé sa réaction très intéressante et je l'ai notée ! Par contre, un peu plus tard, son chum a annoncé qu'il allait à la chasse aux «pardrix », mais lui, il n'a pas eu la moindre réplique ! Un peu perplexe, je me suis dit : «Comment cela se fait-il que lui puisse utiliser cette ouverture, mais pas moi ? » J'ai donc tiré la conclusion suivante : «Ça doit être seulement une voyelle qui s'ouvre quand on est en couple!»

En somme, les expériences vécues par LMF dans cette deuxième partie du récit laissent voir, selon les termes de Celce-Murcia (2007), que c'était des éléments liés à la compétence socioculturelle qu'il désirait principalement parfaire. À son arrivée au Saguenay, bien qu'il ait initialement remis en question sa compétence à l'oral en français, on remarque qu'il ne voulait pas se contenter du simple statut de locuteur bilingue, mais qu'il voulait surtout s'intégrer de manière à être accepté comme usager compétent et légitime de la communauté linguistique saguenéenne. C'était cette volonté d'intégration totale qui guidait ses efforts dans les dernières étapes du développement de sa compétence de communication à l'oral.

\section{Facteurs semblant promouvoir le développement des composantes du modèle de Celce-Murcia (2007)}

On notera dans le récit de LMF que son expérience de développement de sa compétence de communication à l'oral ne correspondait pas intégralement à toutes les composantes du modèle de Celce-Murcia (2007) et n'a pas eu lieu de façon linéaire, l'apprenant accordant plus d'importance à certaines composantes qu'à d'autres. De plus, la lecture du récit de LMF permet de constater que le développement de sa compétence à communiquer en français a été principalement favorisé par des efforts constants et par des stratégies qu'il a mises en place. En ce sens, LMF semblait posséder plusieurs traits du « bon apprenant de langue » (Rubin, 1975, actualisé par Griffiths, 2008), à savoir une motivation soutenue et autorégulée, un style cognitif indépendant du champ, de grandes habiletés métacognitives et une autonomie accrue.

Quelques mots maintenant sur les facteurs internes et externes qui semblent avoir favorisé le développement des compétences évoquées dans le récit, à savoir les compétences linguistique, formulaïque, interactionnelle et socioculturelle.

\section{Facteurs semblant influencer le développement de la compétence linguistique}

D'après le récit de LMF, c'était principalement grâce à l'enseignement formel reçu en classe (aux États-Unis et en France) que l'apprenant a développé certains aspects de sa 
compétence linguistique. On s'étonne peu d'une telle observation puisqu'il est généralement admis dans les écrits scientifiques que l'enseignement explicite est bénéfique, voire souvent nécessaire à l'apprentissage des formes grammaticales, lexicales ou phonétiques (voir Loewen, 2015). Cependant, on note que le développement de sa compétence linguistique a été modulé par les remises en question liées à l'écart observé entre certaines formes privilégiées en classe et les formes entendues et utilisées dans la vie réelle. Ces écarts entre la norme pédagogique et l'usage courant ont d'ailleurs souvent été critiqués par d'autres apprenants de français (voir notamment If this is French, then what did I learn in school? de Durán et McCool, 2002). Comme cet écart semble poser un défi important lors de l'apprentissage du français, il semblerait important d'informer l'apprenant que les formes présentées en classe n'appartiennent peut-être qu'à l'usage formel, et non à la langue courante utilisée quotidiennement par les locuteurs natifs dans des situations de communication informelles ou spontanées.

\section{Facteurs semblant influencer le développement de la compétence formulaïque}

La contrepartie de la compétence linguistique, selon Celce-Murcia (2007), est la compétence formulaïque. Son importance dans la trajectoire développementale de LMF a peu été évoquée, bien que la recherche récente indique que la connaissance de formules stéréotypées contribue grandement au traitement efficace de la langue (Skehan, 1998) et au développement de l'aisance à l'oral (Hilton, 2008 ; Wood, 2009). Pour sa part, c'était uniquement au début de son apprentissage guidé aux États-Unis que LMF a consacré du temps à l'apprentissage de certaines routines et expressions figées. Comme c'était le cas pour la compétence linguistique, les formules enseignées alors ne correspondaient pas aux usages fréquents et naturels des locuteurs natifs. En ce sens, la recherche sur le français L2 peut suivre avec avantage les traces de recherches sur l'identification des formules préfabriquées les plus fréquentes (p. ex. Hsu, 2014), ceci pour favoriser un meilleur développement de la compétence formulaïque.

\section{Facteurs semblant influencer le développement de la compétence interactionnelle}

Le développement de la compétence interactionnelle a pris une certaine place dans le récit de LMF. On peut faire le constat qu'il a initialement développé sa compétence interactionnelle en contexte formel d'apprentissage où on lui a appris des actes de paroles à mémoriser. Cependant, c'était davantage de façon autonome qu'il a développé cette compétence. LMF s'est montré, en effet, très sensible aux rétroactions explicites et implicites fournies par ses interlocuteurs. Il a ajusté ses stratégies de communication en fonction de ces dernières, notamment ses pauses remplies, et a vérifié l'atteinte de ses objectifs en observant le langage non verbal des locuteurs natifs avec qui il interagissait. C'était notamment grâce à son imitation des phénomènes d'hésitations et de pausologie propres aux locuteurs francophones que LMF a estimé qu'il avait atteint une compétence à l'oral assez élevée pour se considérer comme un locuteur bilingue.

\section{Facteurs semblant influencer le développement de la compétence socioculturelle}

Une grande part du récit de LMF est consacrée à des réflexions qui relevaient du développement de sa compétence socioculturelle. Dès ses premiers contacts avec des 
locuteurs natifs, LMF a montré qu'il possédait non seulement une grande conscience sociolinguistique, mais également une efficacité dans le traitement des données langagières ou, pour reprendre la formulation de Skehan (1998), une compétence de traitement (processing competence). Cette compétence donnait parfois lieu à des situations désagréables qui suscitaient chez lui de la frustration, notamment lorsqu'il remarquait des écarts entre les formes qu'on lui avait apprises et les formes utilisées dans des situations de vie réelle. C'est cependant cette même compétence qui lui a permis de mener sa propre enquête de terrain échelonnée sur plusieurs années pour se documenter sur le français parlé au Saguenay.

Par ailleurs, le facteur qui semblait le plus important pour le développement de cette compétence avait trait au but ultime poursuivi par LMF de s'intégrer à sa communauté d'accueil et d'être reconnu comme un membre légitime de cette communauté. L'atteinte de cet objectif, selon lui, a passé par la maîtrise des phénomènes de variation linguistique locaux. Son apprentissage était guidé par sa capacité d'émettre des hypothèses et sa sensibilité à remarquer les réactions de ses interlocuteurs lorsqu'il testait la validité de ses hypothèses.

En somme, le récit de LMF permet de mettre en lumière certains facteurs qui ont semblé favoriser le développement des composantes de la compétence de communication et ce, à différents moments de son parcours d'apprenant/usager du français. On peut constater que malgré le fait que l'enseignement explicite en salle de classe jouait un rôle important pour l'initier à certains rudiments de la langue française, l'atteinte de son objectif était principalement attribuable à ses caractéristiques individuelles conjuguées à sa volonté de vouloir développer une compétence de communication très élevée qui lui ont permis de progresser vers l'atteinte de son objectif.

\section{Réflexions en guise de conclusion}

Après un rappel de l'évolution de la notion de compétence de communication, le récit d'un apprenant à propos du développement de sa compétence de communication a été présenté. Il s'agissait d'un récit qui témoignait des étapes d'un parcours dominé par une quête du sens et par un important besoin de s'intégrer à la communauté d'accueil.

Décrite dans les termes du modèle de Celce-Murcia (2007), la réflexion de cet apprenant à propos de son développement langagier laissait voir l'apparition graduelle de certains éléments de chaque compétence du modèle, à l'exception de la compétence discursive qui n'a pas été évoquée explicitement par l'apprenant. Il laissait aussi voir la présence de facteurs internes et externes qui venaient réguler le développement de sa compétence à communiquer en français.

Si à partir de la représentation graphique du modèle de Celce-Murcia (2007) on aurait pu s'attendre à première vue à y trouver des sous-composantes symétriques et d'égale importance, le récit de LMF a montré qu'il en était autrement. On peut y voir que le développement de chaque sous-compétence se produisait de manière inégale et progressive et que, selon le cas, certaines composantes étaient mobilisées davantage en fonction des capacités, des caractéristiques et de l'atteinte des objectifs personnels de l'apprenant.

Nous reconnaissons que les observations rapportées dans la présente étude font ressortir le parcours développemental relativement singulier et idiosyncrasique de LMF, un linguiste appliqué préoccupé par la réussite de ses interactions avec des locuteurs natifs dans diverses communautés linguistiques. On s'attendrait, par exemple, à ce que les 
données auto déclarées d'un autre apprenant expert ou même d'un apprenant naïf différeraient, surtout si ces derniers ne partageaient pas les préoccupations communicatives de LMF. Malgré la difficulté de transposer les résultats de cette étude à tout autre apprenant, nous croyons qu'il est par ailleurs possible de dégager des constantes (analytic generalization, Yin, 2013), ou des tendances générales, qui, à notre connaissance, ponctuent le développement de la compétence de communication en L2. Ainsi, nous avançons que l'importance donnée au développement de chacune des composantes de la compétence de communication varierait en fonction de la réalité linguistique et des besoins communicatifs de chaque apprenant/usager. De plus, la décision de développer un aspect de la compétence à communiquer ou un autre se ferait à la fois en fonction de choix personnels, d'exigences communicatives du milieu et de la volonté et de la capacité de l'apprenant/usager de satisfaire ces exigences.

Ainsi, grâce à ce récit, on peut penser que le cheminent vers la compétence de communication se présente comme un système dynamique et non linéaire (tout comme l'avançaient Larsen-Freeman, 1997, 2011, 2015 ; Larsen-Freeman et Cameron, 2008) où l'apprenant est artisan de son parcours et de son progrès (voir Mercer, 2011). Les modèles de compétence de communication présentés en introduction ne correspondent pas entièrement à cette réalité et apparaissent plutôt comme une liste ordonnée de connaissances que l'enseignant doit transmettre et que l'apprenant doit, par conséquent, obligatoirement maîtriser. Les réflexions issues de cette étude de cas nous laissent donc croire qu'il serait peut-être utile d'avoir recours à ces modèles non pas pour tenter de développer une compétence de communication définie entièrement par un enseignant, un plan de cours ou un programme, mais bien une compétence de communication définie également par l'apprenant en fonction de sa réalité linguistique et des besoins communicatifs auxquels il veut répondre.

Dans ce sens, les recherches ont indiqué que les enseignants de L2 consacraient principalement leur temps de classe à développer la compétence linguistique de leurs apprenants (et tout particulièrement la grammaire, voir notamment Loewen, 2015). Cependant, dans le cas de LMF, il se rendait compte que sa compétence de communication à l'oral serait perçue comme étant supérieure dans la mesure où il améliorerait sa compétence interactionnelle et que sa compétence socioculturelle se rapprocherait de la norme locale. À cet égard, on aurait certainement intérêt à faire preuve de plus de créativité pédagogique pour faire vivre aux apprenants des expériences d'apprentissage en classe qui sont reliées à la communication naturelle, qui s'éloignent parfois d'un français normatif, et qui placent l'apprenant au cœur de sa propre réalité des échanges langagiers et culturels de tous les jours. Pour ce faire, il serait essentiel de rappeler que le développement de la compétence à l'oral va bien au-delà des exercices de grammaire. En fait, selon les expériences de LMF, ce développement «... . est richement complexe et soumis à toute une gamme de facteurs à la fois linguistiques et psychosociaux dont l'importance varie non seulement en fonction du contexte d'apprentissage, mais surtout en fonction de la réalité et du désir de l'apprenant. »

La correspondance devrait être adressée à Leif French.

Courriel : french@shsu.edu 


\section{Notes}

${ }^{1}$ Le terme compétence de communication (communicative competence) avait été utilisé par d'autres chercheurs tels que Campbell et Wales (1970) et Slobin et coll. (1967) avant que l'expression ne soit associée à Hymes (1972) dans son écrit fondateur On Communicative Competence. Une précision intéressante à ce sujet est cependant fournie par Hymes (1984) dans un texte publié en français.

${ }^{2}$ Voir Leung (2005) pour une revue critique de l'écart trouvé entre les modèles de compétence de communication en pédagogie des langues et les notions mises de l'avant par Hymes (1972). 


\section{Références}

Bachman, L. F. et Palmer, A. S. (1996). Language testing in practice: Designing and developing useful language tests (vol. 1). Oxford, Royaume-Uni : Oxford University Press.

Bachman, L. F. et Palmer, A. S. (2010). Language assessment in practice: Developing language assessments and justifying their use in the real world. Oxford, RoyaumeUni : Oxford University Press.

Campbell, R. et Wales, R. (1970). The study of language acquisition. New Horizons in Linguistics, 242-260.

Canale, M. et Swain, M. (1980). Theoretical bases of communicative approaches to second language teaching and testing. Applied Linguistics, 1(1), 1-47.

Celce-Murcia, M. (2007). Rethinking the role of communicative competence in language teaching. Dans A. Soler et M. S. Jordà (dir.), Intercultural language use and language learning (p. 41-57). Dordrecht, Pays-Bas : Springer.

Celce-Murcia, M., Dörnyei, Z. et Thurrell, S. (1995). Communicative competence: A pedagogically motivated model with content specifications. Issues in Applied Linguistics, 6(2), 5-35.

Chevrot, J.-P., Nardy, A. et Barbu, S. (2011). Developmental dynamics of SES-related differences in children's production of obligatory and variable phonological alternations. Language Sciences, 33(1), 180-191.

Chomsky, N. (1957). Syntactic structures. The Hague, Pays-Bas : Mouton.

Chomsky, N. (1965). Aspects of the Theory of Syntax. Cambridge: M.I.T. Press.

Conseil de 1'Europe. (2001). Cadre européen commun de référence pour les langues. Paris, France : Didier.

Durán, R. et McCool, G. (2002). If this is French, then what did I learn in school? The French Review, 77, 288-299.

Griffiths, C. (2008). Lessons from good language learners. Cambridge, Royaume-Uni : Cambridge University Press.

Guérin, E. (2011). Sociolinguistique et didactique du français : une interaction nécessaire. Le français aujourd'hui (3), 139-144.

Hilton, H. (2008). The link between vocabulary knowledge and spoken L2 fluency. Language Learning Journal, 36(2), 153-166.

Hsu, W. (2014). The most frequent opaque formulaic sequences in English-medium college textbooks. System, 47, 146-161.

Hymes, D. (1967). Models of the interaction of language and social setting. Journal of social issues, 23(2), 8-28.

Hymes, De.(1972). On communicative competence. Dans J.B Pride \& Holmes, J. (Dir.) Sociolinguistics: Selected Readings (pp. 269-293). Harmondsworth: Penguin.

Hymes, D. H. (1984). Vers la compétence de communication. Paris, France : Hatier-Crédif.

Larsen-Freeman, D. (1997). Chaos/complexity science and second language acquisition. Applied Linguistics, 18(2), 141-165.

Larsen-Freeman, D. (2011). A complexity theory approach to second language development/acquisition. Dans D. Atkinson (dir.), Alternative approaches to second language acquisition (vol. 48-72). London, Royaume-Uni/New York, NY : Routledge. 
Larsen-Freeman, D. (2015). Complexity theory. Dans B. Van Patten et J. Williams (dir.), Theories in second language acquistion ( $2^{\mathrm{e}}$ éd., p. 227-244). New York, NY : Routledge.

Larsen-Freeman, D. et Cameron, L. (2008). Complex systems and applied linguistics. Oxford, Royaume-Uni : Oxford University Press.

Lave, J. et Wenger, E. (1991). Situated learning: Legitimate peripheral participation. Cambridge, Royaume-Uni : Cambridge University Press.

Leung, C. (2005). Convivial communication: Recontextualizing communicative competence. International Journal of Applied Linguistics, 15(2), 119-144.

Loewen, S. (2015). Introduction to instructed second language acquisition. New York/London: Routledge.

Mercer, S. (2011). Understanding learner agency as a complex dynamic system. System, 39(4), 427-436.

Moirand, S. (1982). Enseigner à communiquer en langue étrangère. Paris : Hachette.

Rubin, J. (1975). What the "good language learner" can teach us. TESOL quarterly, 9(1), 41-51.

Skehan, P. (1998). A cognitive approach to language learning. Oxford, Royaume-Uni : Oxford University Press.

Slobin, D. I., Ervin-Tripp, S. M., Gumperz, J.J., Brukman, J., Kernan, K., Mitchell, C. et Sross, B. (1967). A field manual for cross-cultural study of the acquisition of communicative competence. Berkely, CA : University of California.

Wood, D. (2009). Effects of focused instruction of formulaic sequences on fluent expression in second language narratives: A case study. The Canadian Journal of Applied Linguistics, 12(1), 39-57.

Yin, R. K. (2013). Case study research: Design and methods ( $5^{\mathrm{e}}$ éd.). Los Angeles, CA : Sage. 


\section{Annexe : Récit intégral de LMF}

\section{Partie I : La compétence de communication : de débutant à apprenant avancé}

Prise de conscience 1 : « Ce qu'on apprend n'est pas toujours ce qu'on entend ! " Au tout début de mon apprentissage, notre enseignante (une Française du sud de la France) nous faisant répéter continuellement des énoncés basés sur un acte de parole. Par exemple elle nous disait : "Bonjour Classe! Comment allez-vous?" — phrase à laquelle nous répondions, comme de petits robots, "Je vais bien! Et vous? " Ou bien, nous produisions d'autres énoncés stéréotypés auprès de notre enseignante ou de nos confrères. Par exemple : "Comment vous appelez-vous? 》 ou "Comment t'appelles-tu? " Une question à laquelle on répondait rapidement et avec fierté : «Je m’appelle...» Il y avait aussi des exercices de répétitions incessants pour travailler non seulement la «bonne » prononciation, mais aussi pour nous sensibiliser à l'ordre des adjectifs en français. Donc, toute la classe chantait souvent en cœur : "Un crayon! Bleu... un crayon bleu! Gros... un gros crayon! Un gros crayon bleu!»

Par contre, vous pouvez vous imaginer la totale déception linguistique vécue lors de notre première activité culturelle au Consulat de France à San Francisco, quand les jeunes étudiants français en échange nous tendaient chaleureusement la main en disant : "Salut, ça va? C'est quoi ton nom? ", ou bien la confusion totale à une question simple posée par l'animateur de l'activité : "Toi, t'es dans quel bus? Le gros bus jaune? » Question à laquelle on se demandait s'il fallait répondre en précisant: «Oui, le bus jaune, le gros bus, le gros bus jaune».

Quelques années plus tard, lors d'un passage à l'Université d'Angers, tout fier d'être inscrit au cours de phonétique avec des locuteurs natifs, je travaillais très fort afin d'assimiler toutes les nuances acoustiques de la langue française. Surtout, je passais des heures au laboratoire de langue pour bien percevoir et bien réaliser la distinction phonétique entre la première personne au singulier au futur et celle du conditionnel, comme dans je prendrai/e/versus je prendrais/ $/ \varepsilon /$.

Par contre au bout d'un an, j'ai dû faire un constat curieux et décourageant. Même dans le Val de Loire, l'endroit où le français serait, semble-t-il, le plus «pur » au monde, je n'étais toujours pas en mesure de percevoir une telle distinction dans le langage spontané de tous les jours. Je me questionnais sérieusement : «Est-ce que je souffrais d'un déficit cognitif de la perception sévère ou était-il possible que cette nuance phonétique soit fictive ou purement théorique? » Ah... quoi faire avec l'apprentissage ?!

\section{Prise de conscience 2 : «À l’oral, les hésitations ou le silence ne sont pas} bons! » Tout au long de mon apprentissage, à l'aide d'une personnalité plus ou moins extrovertie, j'ai profité de chaque occasion, peu importe la situation, pour entrer en communication avec le locuteur natif. Cependant, j'ai vite découvert que les échanges verbaux n'étaient pas toujours agréables et qu'ils pouvaient même 
s'avérer douloureux parfois! Devant mon interlocuteur, je prenais le temps de bien conceptualiser mes idées et ensuite de bien formuler mon énoncé. Pourtant, lors d'échanges verbaux, mon énoncé, bien que construit soigneusement, était souvent accompagné de silence et d'hésitations, et cela provoquait parfois des réponses/réactions complètement inattendues : les gens se penchaient vers moi avec un regard un peu confus, une grimace signalant de l'irritation, de l'impatience ou bien une totale perplexité ! Mon Doux... je me demandais des fois, dans l'effort de sculpter mon identité linguistique en français, si je n'allais pas en même temps développer un trouble de la personnalité ! La gloire de la victoire est finalement arrivée ! Toujours époustouflé par les réactions imprévues de mon interlocuteur, je me suis embarqué dans quête linguistique afin de réduire et de, potentiellement éradiquer le silence et les hésitations irrégulières de mon parler. Conclusion préliminaire de l'enquête — c'était un exploit impossible ! Par contre, une découverte valant de l'or a émergé de cette quête : une nouvelle stratégie de communication me permettait de briser le silence et de camoufler les hésitations. Celle-ci consistait tout simplement à saupoudrer ma parole d'interjections lexicales stratégiques comme : ben....bon ben....t'sais ou bien à utiliser la petite pause euh, mais - attention — soigneusement réalisée à la française comme euh et non pas umm ... ou ahh...!

Quelques années plus tard au Crédit Lyonnais où je travaillais comme traducteur pour un emploi d'été, il y a eu une percée dans ma vie d'apprenant soudainement, je me suis rendu compte que lorsque je communiquais avec les clients, ils ne se penchaient plus que rarement vers moi avec ces fameuses grimaces de confusion et d'incertitude que j'avais pris l'habitude de voir chez mes interlocuteurs. Je me suis donc dit avec de la conviction : "C'est en plein ça! Il faut maîtriser le phénomène de pausologie et d'hésitations pour paraître compétent à l'oral en français! Enfin, j'avais atteint le statut de membre distingué du Club des bilingues, celui qui semblait être certifié ou, tout du moins, approuvé par les locuteurs natifs! Quelle réalisation!

\section{Partie II : La compétence de communication : l'usager compétent et son rendez-vous avec la variété québécoise}

Prise de conscience 3 : « Le français parlé au Québec bat au rythme d'une chanson bien différente. " Après quelques années en Europe, je me suis installé dans la belle province de Québec pour poursuivre mes études supérieures. Lors de mon arrivée au Saguenay, appelé de manière populaire le "Royaume du nord", je débarque dans le petit aéroport de Bagotville et me dirige au comptoir de la location d'autos (et non — bizarrement — — la "location de voitures"). Là, un monsieur d'un certain âge m'attendait avec un sourire chaleureux : "Bonhour Cher !" Pas trop certain ni de la prononciation de "bonhour" ni de la signification de "Cher", je ne me laisse pas déconcerter par l'incertitude lexicale-phonologique et je lui adresse un "Bonjour Monsieur" bien articulé ! Ensuite, il se tourne vers moi et en me regardant au-dessus de ses petites lunettes d'un air curieux, il dit : "Cou'don! Qu'esse tsu vient faire dans c'coin icitte?!' Je suis resté bouche bée !!!! Grâce à son intonation montante, je savais qu'il venait de me poser une question, mais, à ma 
grande surprise, je n'avais pu capter que deux mots : "faire" et "coin". Je commençais sérieusement à remettre en question mon statut au Club des bilingues - comment se faisait-il que ce cher monsieur me comprenne sans problème alors que je pouvais à peine le comprendre ? Après tout, la langue de communication était le français, n'est-ce pas?

Mon dilemme de compréhension devait cependant se poursuivre... ! À peine cinq minutes plus tard, devant le kiosque d'autos, je demande à un chauffeur de taxi comment me rendre au centre-ville de Chicoutimi. Muni d'une mine de connaissances couronnées par l'Académie française et le Grevisse, je planifie soigneusement ma phrase pour qu'elle sonne précise, compétente et intelligible : "S'il vous plait, pourriez-vous m'indiquer où se trouve le boulevard Talbo?" Le chauffeur a hésité un instant : Ah... tsu veux dire le boul'vard (ord) Talbo(t)!

"Garde! c'st ben facile... tsu vas tout drète-là ! Pis.. tsu fais un ptsi boutte... pis tsu vires à gauche-là ! Pis, là ! là ! Tsu fais un autr' boutte un peu plus loin... pis tsu va l'voér" À ma grande déception, je n'ai rien compris cette fois-là !! Tout ce je pouvais déchiffrer de cet échange c'était un enchainement de pi-tsu-là, pi-tsu-là, boutte! pi-tsu-là, boutte! En fait, Il a fallu deux jours de recherche dans les ouvrages de dialectologie avant de bien saisir le sens de "boutte" et de comprendre que le bourdonnement des sons ( $t s u, t s u, d z u$ ) était mon premier cours 101 sur le phénomène de l'affrication au Québec.

Les mois et même les années qui suivirent ces tout premiers contacts avec la variété québécoise furent continuellement remplis de nouvelles expériences riches en matière linguistique et sociolinguistique. Certaines de ces expériences eurent un caractère particulièrement marquant. Par exemple, je me rappelle que mon premier voisin s'appelait " $L u c$ ", mais lorsque sa femme lui criait après dans le garage, elle relâchait tellement la voyelle/y/de Luc que j'ai cru sincèrement, pendant quelques mois, que son nom était "Look" comme en anglais. J'ai bien ri en pensant que cela devait être un nom exotique par ici !

Il y avait aussi mes sorties de pêches où j'ai fait la découverte d'une drôle de forme linguistique. Par exemple, mes "chums" de pêche avaient l'habitude d'employer fréquemment "tu" ou "ti" dans les questions comme "Ça s'peut-tsu?, Ça mordtsu?, T'as-tsu une bière ?" ou encore "Ton couteau, ça coupe-tsi comme y faut?". J'étais très intrigué par la complexité linguistique de ce phénomène et je me suis donc mis à créer et à pratiquer mes propres phrases avec $t s u$.

Un jour, une technicienne en informatique me demande mon numéro de matricule pour remplir une demande et, impatient de démontrer à mon entourage ma compétence de l'emploi de "tu", je lui ai rapidement répondu : "Je sais-tsu moé ?". Sa réponse fut complètement inattendue: "Ah, Monsieur est un peu baveux à matin! 'Évidemment, dans tout mon effort de maîtriser l'emploi structural de la petite particule "tu", j'étais malheureusement passé à côté de certaines nuances pragmatiques assez importantes! 
Prise de conscience 4 : « Je m'aligne-tu au français du Québec et de ma région? » Un moment clé qui a profondément influencé mon identité comme bilingue vivant et travaillant au Québec a été le jour où j'ai pris la décision de m'abandonner complètement à la langue telle qu'elle se parle et qu'elle se vit au Québec. C'était après une sortie de chasse au Lac-Saint-Jean et un ami et moi étions dans un café en train de discuter de la meilleure façon d'installer les "appelants" dans un champ (c.-à-d., les leurres en plastique servant à attirer le gibier). À un moment de la conversation, il m'a arrêté en riant: "T'es donc ben drôle! Pourquoi tu dis tout le temps 'applon'? Nous-autres, on dit pas ça de même! C'est un 'appelin'!' Quelle intervention puissante provenant d'un ami forestier nonlinguiste! Mais aussi quelle révélation personnelle! Dans la petite phrase de mon ami “nous-autres, on dit pas ça!”, on m'avait très curieusement placé à l'extérieur du pronom "nous-autres" et, pourtant, cela faisait déjà plus d'un an que je restais au Québec. Est-ce que je n'étais pas intégré ?!

Jusque-là, je n'avais jamais vraiment considéré à quel point je dépendais de connaissances linguistiques basées sur l'instruction formelle de la langue et/ou sur la norme établie par l'Académie Française pour construire mon discours à l'oral. En fait, pour moi, ces connaissances formelles faisaient partie intégrale de mon identité, de mon existence bilingue. Comment allais-je faire pour atténuer cette dépendance si enracinée dans mon identité linguistique pour pouvoir être inclus dans le "nousautres" de mon ami ? Toutes sortes de questions me sont vite venues à l'esprit : "Est-ce même possible d'être inclus ? Si oui, sur quels indices de l'oral devrais-je me baser pour faire partie du club des 'nous - autres'? Après tout, ni l'Académie française, ni l'Office de la française au Canada, ne semblaient être trop certains quant au chemin à prendre pour obtenir un abonnement au 'Club des nous — autres' au Québec.

Je me suis donc embarqué dans une aventure personnelle à grande échelle pour découvrir la formule magique pour accéder au 'Club des nous-autres'. J'ai porté une attention particulière au discours de tout le monde que je côtoyais. En fait, je suis devenu un véritable laboratoire linguistique mobile et je testais constamment mes hypothèses en fonction de mes observations minutieuses. J'ouvrais certaines voyelles pour être certain de bien réaliser les prononciations comme 'chal $/ \varepsilon /$ ' (chalet), ' $1 / \varepsilon /$ ' (lait), ou bien 'pa' (pas) et 'po' (le gros 'a' dans pas) et ensuite je lisais attentivement la réaction de mon interlocuteur ou je leur demandais carrément leur impression de mon usage. Lorsque je ressentais une impression négative ou un jugement quelconque, j'en prenais note mentalement.

Je dois avouer qu'avec ma méthodologie, cela n'a pas toujours été évident de bien discerner la norme d'usage. Par exemple, un jour, j'ai lâché un gros 'MARCI!' devant une amie et sa réaction a été instantanée et tranchante : 'T'sais... c'est vraiment pas beau de dire ça! C'est les gens peu scolarisés de rang qui disent ça!' J'ai trouvé sa réaction très intéressante et je l'ai notée ! Par contre, un peu plus tard, son chum a annoncé qu'il allait à la chasse aux 'pardrix', mais lui, il n'a pas eu la moindre réplique! Un peu perplexe, je me suis dit : 'Comment cela se fait-il que lui 
puisse utiliser cette ouverture, mais pas moi ? J'ai donc tiré la conclusion suivante : 'Ça doit être seulement une voyelle qui s'ouvre quand on est en couple!'

Cela fait maintenant plus de vingt ans que cette fameuse aventure est en cours. Sincèrement, c'est difficile pour moi de dire à quel point je fais partie du Club des 'nous-autres', mais ce qui est très clair c'est que je m'identifie, de tout mon cœur, au français du Canada, du Québec et surtout du Saguenay-Lac-Saint-Jean! En fait, tout récemment, lors d'une soirée entre amis, je parlais de mon enfance dans la région des vignobles en Californie et un de mes amis s'est mis à rire : 'À savoir que t'est d' c'coin-là... c'a pas de bon sens! T'es quasiment comme nous!' Ah, l'emploi du 'quasiment' dans sa phrase en dit très long, mais cela signifie quoi au juste? Pour moi, c'est le symbole d'un long voyage fascinant qui n'est pas fini, un voyage qui témoigne du fait que le développement de la compétence à l'oral est richement complexe. C'est un développement qui est soumis à toute une gamme de facteurs à la fois linguistiques et psychosociaux dont l'importance varie non seulement en fonction du contexte d'apprentissage, mais surtout en fonction de la réalité et du désir de l'apprenant. 